\title{
Enhanced pinning of superconducting vortices by magnetic vortices
}

\author{
A. Hoffmann,,${ }^{1,2, *}$ L. Fumagalli, ${ }^{3}$ N. Jahedi ${ }^{3}$ J. C. Sautner, ${ }^{3}$ J. E. Pearson, ${ }^{1}$ G. Mihajlović, ${ }^{1}$ and V. Metlushko \\ ${ }^{1}$ Materials Science Division, Argonne National Laboratory, Argonne, Illinois 60439, USA \\ ${ }^{2}$ Center for Nanoscale Materials, Argonne National Laboratory, Argonne, Illinois 60439, USA \\ ${ }^{3}$ Electrical and Computer Engineering, University of Illinois at Chicago, Illinois 60612, USA
}

(Received 16 November 2007; revised manuscript received 15 January 2008; published 28 February 2008)

Periodic arrays of magnetic structures are well known to give rise to commensurate pinning of superconducting vortices in adjacent superconducting films. In this work we compare the pinning effects due to magnetic dots with either single-domain or magnetic-vortex magnetization configuration. We observe a clear correlation between the magnetoresistance in the superconductor and the magnetization configuration of the magnetic dots indicating that the pinning of superconducting vortices is strongly enhanced for the magnetic vortex state. The origin of this enhanced pinning is due to the locally larger stray magnetic fields produced by the magnetic vortex cores.

DOI: 10.1103/PhysRevB.77.060506
PACS number(s): 74.25.Fy, 74.25.Qt, 75.70.Kw
Although the use of periodic magnetic structures for pinning of superconducting vortices has already been suggested in the early $1970 \mathrm{~s},{ }^{1}$ only during the last two decades has it been possible to fabricate hybrid structures of superconductors and ferromagnets with well controlled lateral dimensions necessary to observe commensurate pinning effects. ${ }^{2,3}$ Since then periodic magnetic structures have been used to tune vortex dynamics in many various ways, such as elastic transitions due to geometrical distortions of the vortex lattice with rectangular pinning arrays ${ }^{4}$ and rectifying vortex motion for noncircular magnetic structures. ${ }^{5-7}$ At the same time the influence of the magnetization state on the vortex pinning properties has been extensively studied. ${ }^{8-12}$ In particular, it has been shown that a perpendicular magnetization component can give rise to asymmetric pinning depending on the relative orientation of the magnetization with respect to the magnetic flux of the superconducting vortices ${ }^{8,12}$ and that pinning effects are generally more pronounced for magnetic structures in a single-domain vs multidomain state. ${ }^{9}$

The same advancement in lithographic fabrication of submicrometer magnetic structures that enabled the investigation of superconducting vortex pinning also paved the way for the observation of magnetic vortices in circular magnetic structures. ${ }^{13-16}$ Namely, for small enough circular magnetic structures the magnetostatic energies may stabilize a vortex state at remanence, such that the magnetization curls up along the edges of the circular structure in order to reduce lateral stray fields. Concurrently, the hysteresis loop can be characterized by two critical fields for vortex nucleation and annihilation (see Fig. 1). In contrast, for hysteresis loops dominated by domain wall motion there is generally only one critical field due to either domain wall nucleation or domain wall depinning. ${ }^{17}$ While the magnetization of the vortex state remains mostly oriented in the plane of the magnetic dot, there is, at the vortex center, a singularity where the magnetization points out of plane. ${ }^{14}$ The size of this socalled vortex core is given by the magnetic exchange length; thus it is typically a few $\mathrm{nm}$ in diameter. ${ }^{18,16}$

In this work, we utilized periodic arrays of magnetic dots to study how the formation of magnetic vortices influences the pinning of superconducting vortices. In contrast to earlier investigations focusing on single- vs multidomain states, ${ }^{9}$ we find that the formation of an inhomogeneous magnetization state, i.e., a magnetic vortex, gives rise to more pronounced pinning effects. This result can be explained by the generation of a strong local perpendicular magnetic stray field in the presence of the magnetic vortices.

Square arrays of submicrometer, circular permalloy $\left(\mathrm{Ni}_{80} \mathrm{Fe}_{20}\right)$ dots with $600 \mathrm{~nm}$ diameter, $25 \mathrm{~nm}$ thickness, and $1 \mu \mathrm{m}$ periodicity (see inset of Fig. 1) were fabricated on $\mathrm{Si}(100)$ substrates via standard $e$-beam lithography, $e$-beam evaporation, and liftoff processes. ${ }^{19}$ Room-temperature magnetometry measurements with a superconducting quantum interference device (SQUID) show clear magnetic vortex behavior, as shown in Fig. 1, with a well-defined vortex nucle-

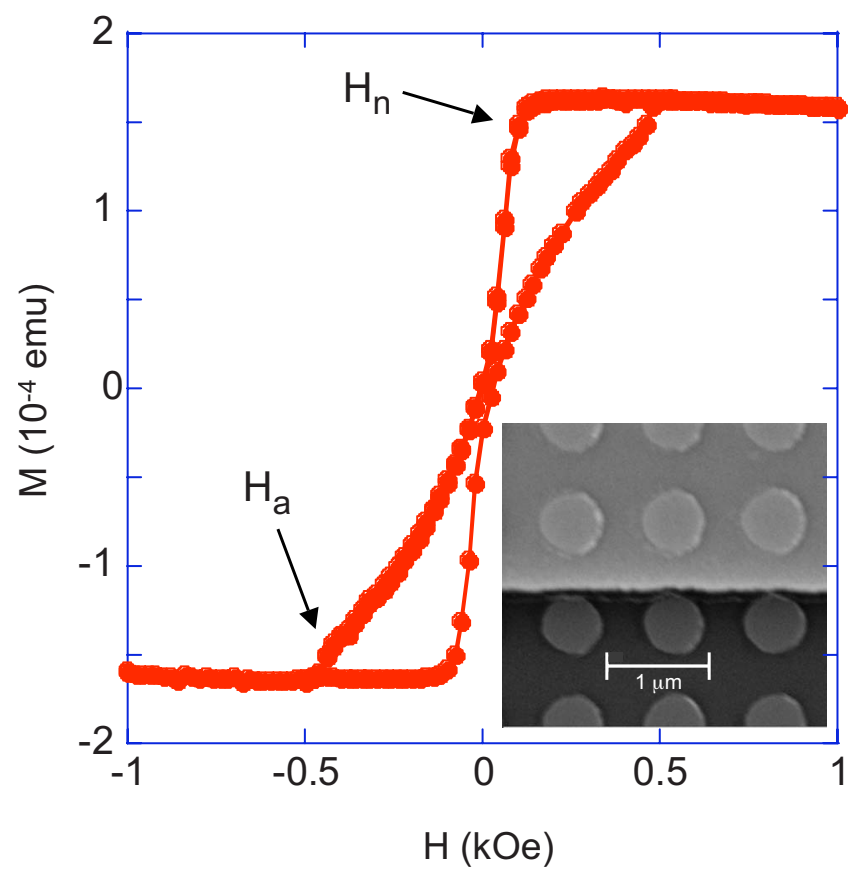

FIG. 1. (Color online) Magnetic hysteresis loop of the magnetic dots measured at $300 \mathrm{~K}$ with in-plane $\left(\alpha=90^{\circ}\right)$ magnetic fields. The inset shows a scanning electron micrograph of the magnetic dot array, and the $\mathrm{Nb}$ stripe covering the top portion of the array. 

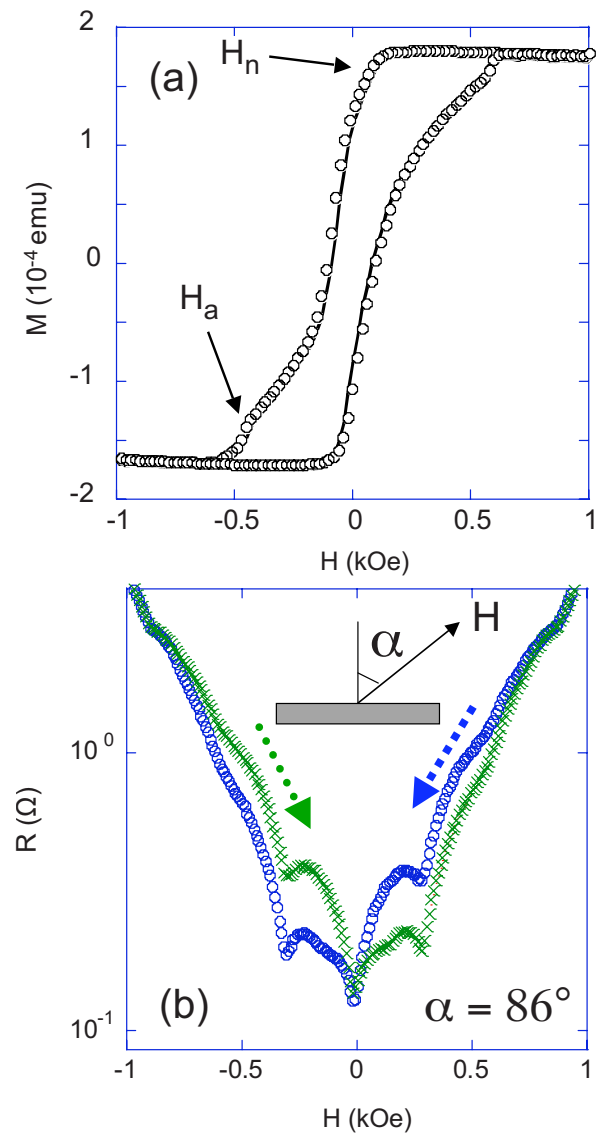

FIG. 2. (Color online) (a) Magnetic hysteresis loop of the magnetic dots measured at $8.27 \mathrm{~K}$ with in-plane $\left(\alpha=90^{\circ}\right)$ magnetic fields. (b) Magnetoresistance of the $\mathrm{Nb}$ measured at $8.27 \mathrm{~K}$ with the magnetic field applied at $\alpha=86^{\circ}$ (see inset) and the maximum fields exceeding the annihilation fields. Open symbols are for decreasing fields, while crosses are for increasing fields.

ation field $H_{n}$ and vortex annihilation field $H_{a}$. In order to investigate through magnetotransport measurements the effects of magnetic vortices on superconducting vortex pinning we fabricated a four-probe measurement bridge $(100 \mu \mathrm{m}$ wide and $500 \mu \mathrm{m}$ long) of $100 \mathrm{~nm}$ thick $\mathrm{Nb}$ on top of the permalloy dot array via optical lithography, sputter deposition, and liftoff. The four-point dc magnetoresistance of these structures was measured in a helium cryostat equipped with a $60 \mathrm{kG}$ superconducting magnet and a rotating sample probe so that the magnetic field can be applied at a variable angle $\alpha$ with respect to the surface normal [see inset of Fig. 2(b)].

The field dependence of the resistance measured at 8.27 K (just below $T_{c}=8.32 \mathrm{~K}$ ) is shown in Fig. 2(b) for both increasing and decreasing magnetic fields with $\alpha=86^{\circ}$. There are clear periodic minima in the resistance indicating coherent vortex pinning. For varying $\alpha$ the periodicity $\Delta H$ between adjacent peaks scales with the perpendicular field component $H \cos (\alpha)$, indicating that only the perpendicular field component is relevant for the formation of the superconducting vortices, which is consistent with previous observations. ${ }^{3}$ Note that for $\alpha=86^{\circ}$ the in-plane component is $99.8 \%$ of the total applied magnetic field, while the out- of-plane component is $7.0 \%$. Furthermore the periodicity corresponds to matching fields given by the lattice constant $a$ of the square magnetic dot array, such that $\Delta H \cos (\alpha)$ $=\Phi_{0} / a^{2}$, where $\Phi_{0}=2.07 \times 10^{-7} \mathrm{G} \mathrm{cm}^{2}$ is the quantum of flux. ${ }^{20}$

However, in contrast to earlier investigations, ${ }^{3,4,10}$ the magnetoresistance shows a pronounced hysteresis. A comparison with the magnetic hysteresis of the dots measured by SQUID at the same temperature [see Fig. 2(a)] shows that the hysteretic region of the magnetoresistance coincides with the hysteresis observed in the magnetization of the magnetic dots. In particular, note that for decreasing fields between 0 and $-0.6 \mathrm{kOe}$, i.e., in the presence of magnetic vortices, the resistance is lower than the resistance measured with increasing fields in the same field range. The same behavior is also observed for positive fields. Therefore, the resistance at the first matching field is always higher when the dot is in a quasisaturated state compared to the resistance at the first matching field when the magnetic dot is in the vortex state. This indicates that the pinning of superconducting vortices is enhanced by the presence of a magnetic vortex.

We further investigated this effect by performing minor hysteresis loops with fields below the magnetic vortex annihilation field ( $\left.H_{a} \approx 0.5 \mathrm{kOe}\right)$. As can be seen in Fig. 3(a), the change of magnetization is completely reversible in this field range, which indicates a reversible motion of the magnetic vortex cores. ${ }^{15}$ Furthermore, the magnetoresistance of the $\mathrm{Nb}$ stripe is also completely reversible and the previously observed pronounced asymmetry in the field dependence [see Fig. 2(b)] has vanished. Similar behavior has also been observed for other values of $\alpha$. We therefore conclude that the enhanced pinning of the superconducting vortices is caused by the presence of magnetic vortices in the permalloy dots.

At first thought, this observation that magnetic vortices give rise to enhanced pinning of superconducting vortices is rather surprising. Van Bael et al. investigated the periodic pinning from arrays of rectangular Co structures prepared in either a single-domain or flux-closed two-domain state. They observed that the flux-closed structure showed diminished superconducting vortex pinning and concluded that the stray magnetic fields play an important role for the superconducting vortex pinning. Thus one would expect circular dots to be less effective for superconducting vortex pinning when the magnetic dots are in a magnetic vortex state compared to a single-domain state, since the higher average perpendicular stray fields are generated in the latter case.

However, while the overall external stray magnetic field is reduced, the out-of-plane component of the stray magnetic field is locally strongly enhanced due to the presence of the perpendicularly magnetized core of the magnetic vortex. This can be seen in Fig. 4, where we show the spatial distribution of the magitude of the perpendicular stray field component obtained from micromagnetic simulations using the OOMMF code. ${ }^{21}$ From Fig. 4 it is clear that the magnitude of the perpendicular magnetic field component is strongly enhanced locally, i.e., in the region right above the magnetic vortex core, although its average value within the square area enclosing the dot is lower.

The obvious question is whether the enhanced pinning is due to magnetic interactions between the magnetic vortex 

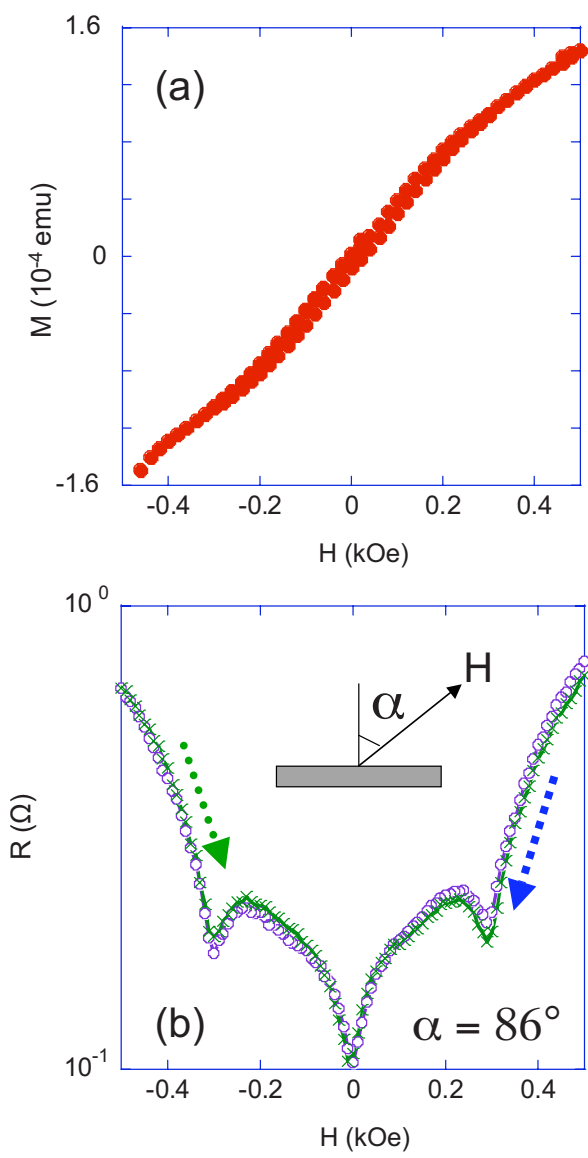

FIG. 3. (Color online) (a) Minor magnetic hysteresis loop of the magnetic dots measured at $8.27 \mathrm{~K}$ with in-plane $\left(\alpha=90^{\circ}\right)$ magnetic fields. (b) Magnetoresistance of the $\mathrm{Nb}$ measured at $8.27 \mathrm{~K}$ with the magnetic field applied at $\alpha=86^{\circ}$ (see inset) and the maximum fields below the annihilation fields. Open symbols are for decreasing fields, while crosses are for increasing fields.

cores and the magnetic flux of the superconducting vortices. To elucidate this question it is worthwhile noting that the minor loop of the magnetoresistance in Fig. 3(b) shows very little asymmetry between positive and negative fields. Since the magnetic vortex state was induced with a tilted magnetic field, the polarization of all magnetic vortex cores should be identical during the nucleation of the magnetic vortices. At the same time the perpendicular field required for switching the vortex core is higher than $1 \mathrm{kOe},{ }^{22}$ and therefore the magnetic vortex core polarity should not change its sign during the minor loop. If there were strong magnetostatic interactions between the magnetic vortex cores and the magnetic flux of the superconducting vortices, one would expect to observe a pronounced asymmetry in the magnetoresistance for positive and negative fields, i.e., when the relative orientation of the superconducting vortex flux with respect to that of the magnetic vortex core changes from parallel to antiparallel. Such asymmetry has been previously observed in systems with perpendicularly magnetized magnetic structures. ${ }^{8}$ The absence of this asymmetry leads to the conclusion that the magnetostatic interactions between magnetic and superconducting vortices are therefore negligible in our samples.
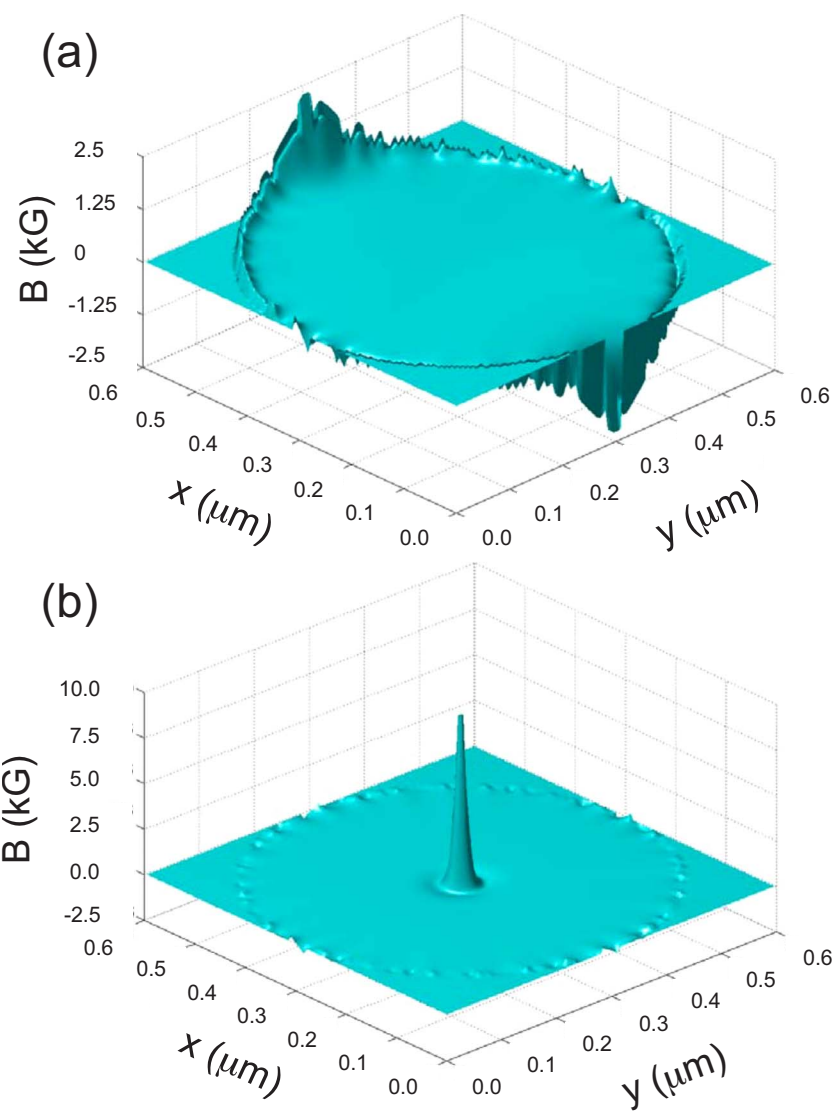

FIG. 4. (Color online) Perpendicular stray field distribution generated by a magnetic dot in (a) a saturated and (b) a vortex magnetization state. The distributions were obtained by micromagnetic simulations.

More likely the strong perpendicular magnetic field of the magnetic vortex core suppresses locally the superconductivity and therefore results in an enhanced pinning of the superconducting vortices. Notice that the magnitude of the stray field of the magnetic vortex cores $[\approx 10 \mathrm{kG}$, see Fig. 4(b)] is much larger than $H_{c 2}$ for temperatures within $20 \%$ of $T_{c}$ of these $\mathrm{Nb}$ films. ${ }^{23}$ Furthermore, the size of the magnetic vortex core corresponds better to the superconducting coherence length $\left(\approx 90 \mathrm{~nm}\right.$ for $\left.T / T_{c}=0.99\right)$, while it is much smaller than the magnetic penetration depth $\left(\approx 800 \mathrm{~nm}\right.$ for $T / T_{c}$ $=0.99) \cdot{ }^{10,18}$ This also supports the idea that magnetostatic interactions are less important than the stray-field mediated proximity effects.

In summary, we have shown that the flux-flow magnetoresistance of a superconducting $\mathrm{Nb}$ film is closely associated with the presence and absence of magnetic vortices in adjacent parmalloy dots. Namely, the formation of magnetic vortices strongly enhances the pinning of superconducting vortices. On the other hand, the magnetoresistance of the $\mathrm{Nb}$ film does not show any asymmetry when the relative orientation of the magnetic flux of the superconducting vortices is changed from parallel to antiparallel with respect to that of the magnetic vortex cores. This suggests that the physical mechanism of the enhanced superconducting vortex pinning 
is not related to magnetostatic interactions but is rather due to the local suppression of superconductivity by highly localized, large out-of-plane stray magnetic fields generated by the magnetic vortex cores.

Recently, Villegas, Li, and Schuller observed a suppression of superconductivity in the presence of magnetic vortices for aluminum thin films covering iron nanodots. ${ }^{24}$ This result is consistent with our explanation of enhanced super- conducting vortex pinning effects caused by magnetic vortices.

This work was financially supported by the U.S. National Science Foundation through Grant No. ECS-0202780 and by the U.S. Department of Energy, Basic Energy Science under Contract No. DE-AC02-06CH1357. *hoffmann@anl.gov

${ }^{1}$ S. H. Autler, Helv. Phys. Acta 45, 851 (1972).

${ }^{2}$ Y. Otani, B. Pannetier, J. P. Nozières, and D. Givord, J. Magn. Magn. Mater. 126, 622 (1993).

${ }^{3}$ J. I. Martín, M. Vélez, J. Nogués, and I. K. Schuller, Phys. Rev. Lett. 79, 1929 (1997).

${ }^{4}$ J. I. Martín, M. Vélez, A. Hoffmann, I. K. Schuller, and J. L. Vicent, Phys. Rev. Lett. 83, 1022 (1999).

${ }^{5}$ J. E. Villegas, S. Savel'ev, F. Nori, E. M. Gonzalez, J. V. Anguita, R. García, and J. L. Vicent, Science 302, 1188 (2003).

${ }^{6}$ A. V. Silhanek, W. Gillijns, V. V. Moshalkov, V. Metlushko, and B. Ilic, Appl. Phys. Lett. 89, 182505 (2006).

${ }^{7}$ A. V. Silhanek, W. Gillijns, V. V. Moshalkov, V. Metlushko, F. Gozzini, B. Ilic, C. Uhlig, and J. Unguris, Appl. Phys. Lett. 90, 182501 (2007).

${ }^{8}$ D. J. Morgan and J. B. Ketterson, Phys. Rev. Lett. 80, 3614 (1998).

${ }^{9}$ M. J. van Bael, K. Temst, V. V. Moshchalkov, and Y. Bruynseraede, Phys. Rev. B 59, 14674 (1999).

${ }^{10}$ A. Hoffmann, P. Prieto, and I. K. Schuller, Phys. Rev. B 61, 6958 (2000).

${ }^{11}$ M. V. Milošević, S. V. Yampolskii, and F. M. Peeters, Phys. Rev. B 66, 174519 (2002).

${ }^{12}$ M. J. van Bael, M. Lange, S. Raedts, V. V. Moshchalkov, A. N.
Grigorenko, and S. J. Bending, Phys. Rev. B 68, 014509 (2003).

${ }^{13}$ R. P. Cowburn, D. K. Koltsov, A. O. Adeyeye, M. E. Welland, and D. M. Tricker, Phys. Rev. Lett. 83, 1042 (1999).

${ }^{14}$ T. Shinjo, T. Okuno, R. Hassdorf, K. Shigeto, and T. Ono, Science 289, 930 (2000).

${ }^{15}$ K. Y. Guslienko, V. Novosad, Y. Otani, H. Shima, and K. Fukamichi, Phys. Rev. B 65, 024414 (2001).

${ }^{16}$ A. Wachowiak, J. Wiebe, M. Bode, O. Pietzsch, M. Morgenstern, and R. Wiesendanger, Science 298, 577 (2002).

${ }^{17}$ S. Chikazumi, Physics of Ferromagnetism (Oxford University Press, New York, 1997).

${ }^{18}$ J. Miltat and A. Thiaville, Science 298, 555 (2002).

${ }^{19}$ J. I. Martín, Y. Jaccard, A. Hoffmann, J. Nogués, J. M. George, J. L. Vicent, and I. K. Schuller, J. Appl. Phys. 84, 411 (1998).

${ }^{20}$ K. Harada, O. Kamimua, H. Kasai, T. Masuda, A. Tonomura, and V. V. Moshalkov, Science 274, 1167 (1996).

${ }^{21}$ URL http://math.nist.gov/oommf

${ }^{22}$ T. Okuno, K. Shigeto, T. Ono, K. Mibu, and T. Shinjo, J. Magn. Magn. Mater. 240, 1 (2002).

${ }^{23}$ A. Hoffmann, Ph.D. thesis, University of California, San Diego, 1999.

${ }^{24}$ J. E. Villegas, C.-P. Li, and I. K. Schuller, Phys. Rev. Lett. 99, 227001 (2007) 